\title{
ORIGINAL ARTICLE \\ Mapping and recombination analysis of two moth colour mutations, Black moth and Wild wing spot, in the silkworm Bombyx mori
}

\author{
$\mathrm{K} \mathrm{Ito}^{1}$, S Katsuma ${ }^{2}$, S Kuwazaki ${ }^{3}$, A Jouraku ${ }^{3}$, T Fujimoto ${ }^{4}$, K Sahara ${ }^{4}$, Y Yasukochi $^{3}, \mathrm{~K} \mathrm{Yamamoto}^{3}$, \\ H Tabunoki ${ }^{1}$, T Yokoyama ${ }^{1}, K_{\text {Kadono-Okuda }}{ }^{3}$ and T Shimada ${ }^{2}$
}

Many lepidopteran insects exhibit body colour variations, where the high phenotypic diversity observed in the wings and bodies of adults provides opportunities for studying adaptive morphological evolution. In the silkworm Bombyx mori, two genes responsible for moth colour mutation, $B m$ and $W s$, have been mapped to 0.0 and $14.7 \mathrm{cM}$ of the $B$. mori genetic linkage group 17; however, these genes have not been identified at the molecular level. We performed positional cloning of both genes to elucidate the molecular mechanisms that underlie the moth wing- and body-colour patterns in $B$. mori. We successfully narrowed down $\mathrm{Bm}$ and Ws to 2-Mb-long and 100-kb-long regions on the same scaffold Bm_scaf33. Gene prediction analysis of this region identified 77 candidate genes in the $B m$ region, whereas there were no candidate genes in the Ws region. Fluorescence in-situ hybridisation analysis in Bm mutant detected chromosome inversion, which explains why there are no recombination in the corresponding region. The comparative genomic analysis demonstrated that the candidate regions of both genes shared synteny with a region associated with wing- and body-colour variations in other lepidopteran species including Biston betularia and Heliconius butterflies. These results suggest that the genes responsible for wing and body colour in $B$. mori may be associated with similar genes in other Lepidoptera.

Heredity (2016) 116, 52-59; doi:10.1038/hdy.2015.69; published online 29 July 2015

\section{INTRODUCTION}

In Lepidoptera, adult body colour patterns are important for sexual selection, mimicry and predator avoidance (Parcham et al., 2007). The wings of insects are believed to be a monophyletic adaptation that allowed the insects to exploit new niches, thereby resulting in rapid diversification. Many studies have investigated the factors that control the wing- and body-colour patterns of butterflies and moths; however, the underlying mechanism still remains unknown. Recently, the genomes and genomic information have been updated for various lepidopteran insects and molecular genetic studies have provided information that is useful for this field of study (International Silkworm Genome Consortium, 2008; Zhan et al., 2011; Heliconius Genome Consortium, 2012; You et al., 2013).

Over 50 body colour mutants have been reported in the silkworm B. mori (Banno et al., 2010). However, most of these mutants correspond to larval body colour variations and few wing- and body-colour variations have been reported in this moth. Five mutants have been reported, that is, Black moth (Bm; Chikushi, 1960), blackstriped pupal wing (bpw; Yamamoto, 1986), melanism ( $m l n$; Hasimoto, 1961), Wild wing spot (Ws; Doira et al., 1981) and white-banded black wing ( $w b$; Kanbe and Nara, 1959). Recently, the $m l n$ mutant, which exhibits a readily distinguishable phenotype in both the larvae and adults, was characterised at the molecular level based on positional cloning and functional analysis (Dai et al., 2010; Zhan et al., 2010). Linkage analysis and genomic studies have shown that Bombyx arylalkamine- $N$-acetyl transferase, the homologous gene (Dat) that converts dopamine into $N$-acetyl dopamine, encodes a precursor of $\mathrm{N}$-acetyl dopamine, sclerotin in Drosophila and it is the gene responsible for $m l n$ (Dai et al., 2010; Zhan et al., 2010). However, other causal genes have not yet been identified.

The Bm mutant has black scales on the body and wings, which contrasts with the white appearance of the wild-type moth (Figure 1a). The gene responsible, $B m$, has been mapped to $0.0 \mathrm{cM}$ in $B$. mori genetic linkage group 17 (Chikushi, 1960) (Figure 1b). The Ws mutant strain exhibits a phenotype where the moth has a spot on the apex of its wing (Figure 1a). The Ws gene has been transferred by introgression from the wild silkworm Bombyx mandarina, which is widely believed to have the same ancestor as the domesticated silkworm B. mori (Goldsmith et al., 2005). This gene has been mapped to $14.7 \mathrm{cM}$ in linkage group 17 and it is linked to the bts (brown head and tail spot) gene (Doira et al., 1981; Banno et al., 2010) (Figure 1b). The Bm and $W s$ phenotypes are both dominant over the wild type. In addition, according to our observations these phenotypes are clearly exhibited in males, whereas it is difficult to distinguish mutant females from the

${ }^{1}$ Department of Science of Biological Production, Graduate School of Agriculture, Tokyo University of Agriculture and Technology, Fuchu, Tokyo, Japan; ${ }^{2}$ Department of Agricultural and Environmental Biology, Graduate School of Agricultural and Life Sciences, The University of Tokyo, Bunkyo-ku, Tokyo, Japan; ${ }^{3}$ Division of Insect Sciences, National Institute of Agrobiological Sciences, Tsukuba, Ibaraki, Japan and ${ }^{4}$ Faculty of Agriculture, Iwate University, Morioka, Iwate, Japan

Correspondence: Dr K Ito, Department of Science of Biological Production, Tokyo University of Agriculture and Technology, 3-5-8 Saiwai-cho, Fuchu, Tokyo 183-8509, Japan. E-mail: katsuito@cc.tuat.ac.jp

Received 20 April 2015; accepted 22 June 2015; published online 29 July 2015 
a

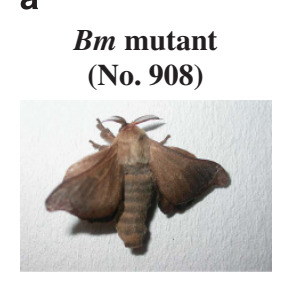

Ws mutant

(u42)



b
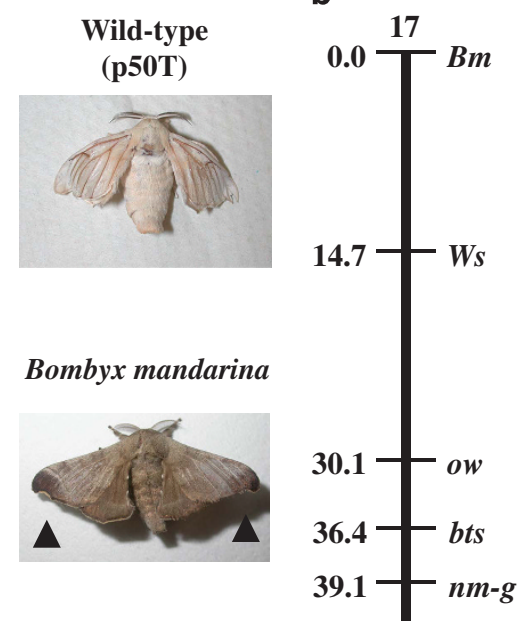

Suc-1

nsd-2

Figure 1 Phenotypes and linkage maps of the $B m$ and $W s$ mutations. (a) Phenotypes of B. mori wild type (p50T), Bm mutant (No. 908), Ws mutant (u42) and B. manderina. Arrowheads indicate the spot at the apex of the wing. (b) Linkage map of group 17. The loci are labelled based on their position in centimorgan units (left) and the locus name (right). Abbreviations: $B m$, Black moth; Ws, Wild wing spot; ow, waxy translucent; bts, brown head and tail spot; $n m-g$, non-molting glossy; nsd-2, nonsusceptibility to DNV-2; Suc-1, sucrase-1 (Banno et al., 2010).

wild type in $\mathrm{BC}_{1}$ individuals. Recently, we succeeded in the positional cloning of four genes responsible for $b t s, n m-g, n s d-2$ and $o w$, which also map to linkage group 17 (Ito et al., 2008, 2009, 2010; Niwa et al., 2010) (Figures $1 \mathrm{~b}$ and 2a). We consider that the genomic information obtained in previous studies may be a useful tool for isolating and identifying $\mathrm{Bm}$ and $\mathrm{Ws}$ mutations.

To better understand the molecular mechanisms that control colour variations in a Lepidoptera, we performed positional cloning and recombination analysis of two genes, that is, $B m$ and $W s$. Based on mapping, we successfully narrowed down the candidate regions of both genes to one scaffold, Bm_scaf33. In addition, recombination analysis between $\mathrm{Bm}$ and $\mathrm{Ws}$, and fluorescence in-situ hybridisation (FISH) analysis demonstrated that chromosome 17 carrying the $\mathrm{Bm}$ gene has inversion in the candidate region. Therefore, recombination between both genes occurred in none of the individuals. Moreover, we found that the candidate regions of both genes shared correspondence with a region associated with wing- and body-colour variations in different lepidopteran species, that is, B. betularia, Heliconius cydno, Heliconius erato, Heliconius melpomene and Heliconius numata (Joron et al., 2006; Kronfost et al., 2006; Papa et al., 2008; Ferguson et al., 2010; van't Hof et al., 2011). These results strongly suggest that the same genes and/or regulatory elements responsible for wing and body colour in Bombyx, Bm and $W s$, may underlie these variants in different Lepidoptera.

In this study, we demonstrate that the genomic context is highly relevant given the orthology in lepidopteran patterning regions and the fact that the Ws mutation appears to influence three nearby genes that do not fall within the 100-kb mapping interval. The apparent involvement of clustered genes in similar processes suggests the existence of a supergene. B. mori is the most advanced model Lepidoptera, thereby facilitating interpretation in a genomic context.

a
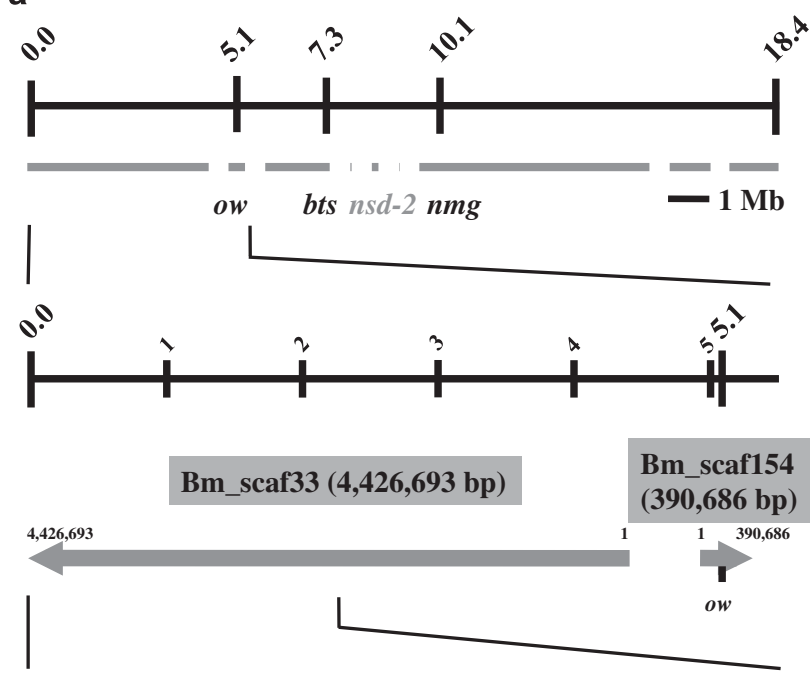

b

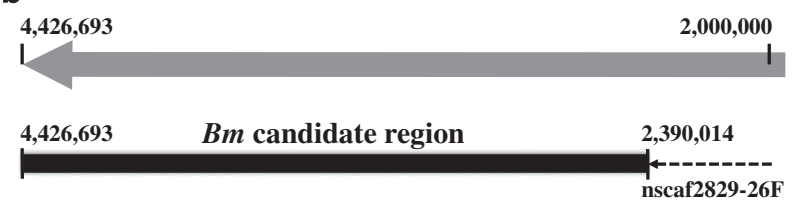

Ws candidate region

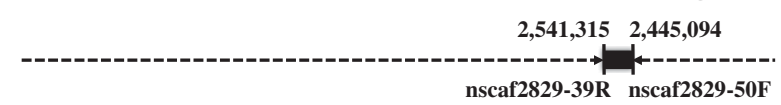

Figure 2 Mapping of the $B m$ and Ws mutations in linkage group 17. (a) Physical map and scaffold of linkage group 17. Black and grey lines indicate the physical map and the scaffold, respectively. The upper and lower figures indicate the whole and upstream regions of linkage group 17, respectively. The upper numbers indicate the positions that correspond to each gene (Ito et al., 2008, 2009, 2010; Niwa et al., 2010). nsd-2 could not be mapped onto the physical map of group 17, because it was located on a non-mapped scaffold (Bm_scaf131). ow was mapped to the Bm_scaf154 (Ito et al., 2009). (b) The narrowed down candidate region on the Bm_scaf33. The dotted arrows indicate the results of the detailed linkage analysis to narrow down the region linked to the $B m$ (upper) and $W s$ (lower) mutations (Table 2). The black boxes are candidate regions of each mutations.

\section{MATERIALS AND METHODS}

\section{Insects}

The $B m\left(B m / B m ;+{ }^{W s} /+^{W s}\right)$ and the $W s\left(+{ }^{B m} /+{ }^{B m} ; W s / W s\right)$ used No. 908 (National Institute of Agrobiological Sciences, Tsukuba, Japan) and u42 (Kyushu University, Fukuoka, Japan), respectively. The wild type $\left(+^{B m} /+^{B m}\right.$; $+{ }^{W_{s}} /+{ }^{W_{s}}$ ) were p50T (University of Tokyo, Bunkyo-ku, Japan) and p50 (Kyushu University) (Figure 1a). $\mathrm{BC}_{1}$ progeny from the cross $\mathrm{p} 50 \mathrm{~T} \times(\mathrm{p} 50 \mathrm{~T} \times$ No. 908) and $\mathrm{p} 50 \mathrm{~T} \times(\mathrm{p} 50 \mathrm{~T} \times \mathrm{u} 42)$ were used for mapping $B m$ and $W s$, respectively. The offspring from the cross $\mathrm{p} 50 \mathrm{~T} \times(\mathrm{u} 42 \times$ No. 908$)$ were used for the recombination analysis between $B m$ and $W s$. All of the silkworm larvae were reared on mulberry leaves at $25^{\circ} \mathrm{C}$.

In the screening of $\mathrm{BC}_{1}$, the $B m$ and $W s$ phenotypes present themselves clearly in males, while mutant females can be hard to distinguish from wild type. Therefore, we only used males in the analysis.

\section{Preparation for genomic DNA and PCR analysis}

DNA was isolated from moth legs using DNAzol (Invitrogen, Carlsbad, CA, USA) according to the manufacturer's protocol. PCR was performed using Ex Taq DNA Polymerase (Takara Bio, Otsu, Japan) and the primer sets are listed 
in Supplementary Table S1. The PCR conditions were as follows: initial denaturation at $94^{\circ} \mathrm{C}$ for 2 min followed by 35 cycles of denaturation at $94{ }^{\circ} \mathrm{C}$ for $15 \mathrm{~s}$, annealing at $60^{\circ} \mathrm{C}$ for $15 \mathrm{~s}$ and extension at $72^{\circ} \mathrm{C}$ for 1 or $3 \mathrm{~min}$ with a final incubation step at $72^{\circ} \mathrm{C}$ for $4 \mathrm{~min}$.

\section{Isolation of total RNA and reverse-transcriptase PCR analysis}

Total RNA was isolated from the forewings of pupae and adults using TRIzol (Invitrogen) according to the manufacturer's protocol. The isolated RNA was reverse transcribed using an Oligo $(\mathrm{dT})_{12-18}$ primer (GE Healthcare, Buckinghamshire, UK) and Ready-to-Go RT-PCR Beads (GE Healthcare), according to the manufacturer's protocol, and the cDNA was then diluted 10-fold before reverse-transcriptase PCR (RT-PCR). RT-PCR was performed using Ex Taq DNA Polymerase, with the primer sets listed in Supplementary Table S1 in the following conditions: initial denaturation at $94^{\circ} \mathrm{C}$ for $2 \mathrm{~min}$ followed by 30 cycles of denaturation at $94^{\circ} \mathrm{C}$ for $15 \mathrm{~s}$, annealing at $60^{\circ} \mathrm{C}$ for $15 \mathrm{~s}$ and extension at $72^{\circ} \mathrm{C}$ for $1 \mathrm{~min}$ followed by a final incubation at $72{ }^{\circ} \mathrm{C}$ for $4 \mathrm{~min}$.

\section{Positional cloning}

Positional cloning of the $\mathrm{Bm}$ and $\mathrm{Ws}$ candidate genes was performed as previously described (Ito et al., 2009). PCR and single-nucleotide polymorphism markers that exhibited polymorphism in the parents were detected at each position on chromosome 17. Mapping was performed using 1861 and $434 \mathrm{BC}_{1}$ progeny with the $B m$ and $W s$ phenotypes, respectively. Candidate genes in the region narrowed by linkage analysis were predicted and annotated using KAIKObase (http://sgp.dna.affrc.go.jp/KAIKObase/), KAIKOBLAST (http://kaikoblast.dna.affrc. go.jp/) and NCBI BLAST (http://blast.ncbi.nlm.nih.gov/Blast.cgi).

\section{Recombination analysis between $\mathrm{Bm}$ and $\mathrm{Ws}$}

Recombination analysis was performed using 1163 male moths obtained by crossing wild-type females with $F_{1}$ males. The progeny could be classified according to four different phenotypes in terms of their body and wing colours: $B m$ type $\left(+^{B m} / B m ;++^{W_{s}} /+^{W s}\right)$, Ws type $\left(+^{B m} /+^{B m} ;+^{W_{s}} / W s\right), B m$ and $W s$ type $\left(+^{B m} / B m ;++^{W_{s}} / W s\right)$, and normal type $\left(+^{B m} /+^{B m} ;++^{W_{s}} /+^{W_{s}}\right)$ (Supplementary Figure S1). However, $B m$ is overdominant to $W s$ phenotype, which made it impossible to discriminate $B m$ and $W s$ type from $B m$ type. Hence, we count the former type together with the latter type. Recombination between $B m$ and Ws occurred in the Bm and Ws types and the normal type, but we judged only from the numbers of the normal type.

\section{FISH analysis}

Bacterial artificial chromosomes (BACs) used for FISH analysis were described by Yasukochi et al. (2006) (Table 1). We selected additional 4D3C and 3C11C BACs for the present study (Table 1). Chromosomes were prepared according to Sahara et al. (1999) and Yoshido et al. (2014). Briefly, female and/or male gonads were dissected from last instar larvae. The cells in the gonads were spread on a glass slide with $60 \%$ acetic acid at $50^{\circ} \mathrm{C}$. The chromosomes were air dried and stored until further use, at $-20^{\circ} \mathrm{C}$ after dehydration with an ethanol series of $70 \%, 80 \%$ and $99 \%$. BAC-FISH analysis was performed as described by Yoshido et al. (2005) and Sahara et al. (2013). Briefly, BAC DNA extracted with a Plasmid Midi kit (Qiagen GmbH, Hilden, Germany) was labeled with fluorochromes (Orange-, Green- and Red-dUTP purchased from Abbott Molecular Inc., Des Plaines, IL, USA, and Cy5-dUTP from GE Healthcare) using a Nick Translation Mix (Roche Diagnostics Inc., Basel, Switzerland) (Table 1). Hybridisation was performed at $37^{\circ} \mathrm{C}$ for 3 days, which was followed by washing with $0.1 \times$ SSC and $0.1 \%$ Triton X-100. Re-probe technique was also used according to Shibata et al. (2009). The FISH preparations were counterstained and mounted with antifade $(0.233 \mathrm{~g} 1,4$ diazabicyclo(2.2.2)-octane, $1 \mathrm{ml} \quad 0.2 \mathrm{~mm}$ Tris- $\mathrm{HCl}, \mathrm{pH} \quad 8.0,9 \mathrm{ml}$ glycerol) containing $0.5 \mu \mathrm{g} \mathrm{ml}^{-1}$ DAPI (4',6-diamidino-2-phenylindole; Sigma-Aldrich, St Louis, MO, USA). Signals were captured with a DFC350FX CCD camera mounted on a DM 6000B microscope (Leica Microsystems Japan, Tokyo, Japan) and processed with Adobe Photoshop CS6J (Adobe, San Jose, CA, USA).

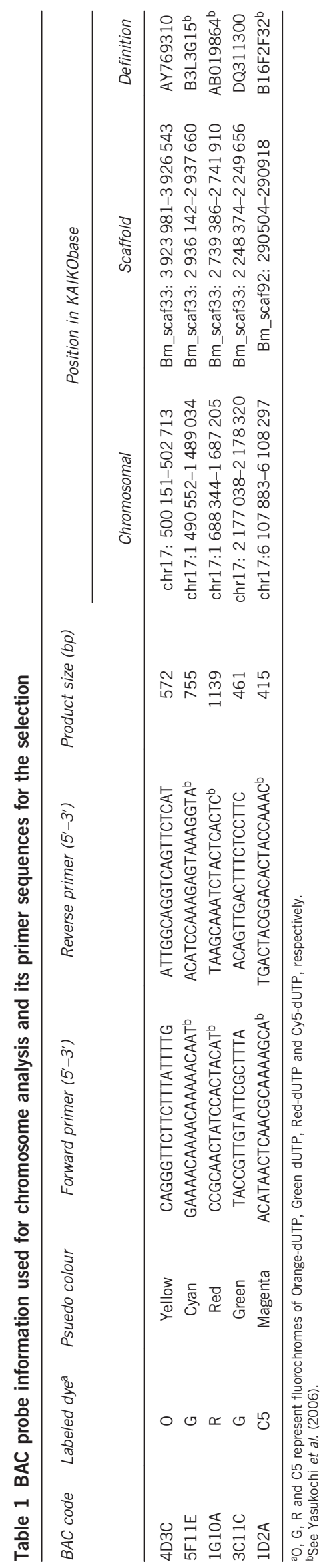




\section{RESULTS}

\section{Mapping of $\mathrm{Bm}$ and $\mathrm{Ws}$}

To identify the genomic regions responsible for $B m$ and $W s$ mutations, we performed genetic linkage analysis referred by the B. mori singlenucleotide polymorphism linkage map (Yamamoto et al., 2008) and genome sequence (International Silkworm Genome Consortium, 2008). The female body colour of $\mathrm{BC}_{1}$ was too faint to allow us to distinguish each phenotype; therefore, we only used males for screening. We mapped the $\mathrm{Bm}$ mutation using $\sim 1800 \mathrm{BC}_{1}$ individuals and narrowed down the Bm-linked region to between 2390014 (nscaf2829-26F) and 4426693 (the downstream terminal of the Bm_scaf33) (Figure 2 and Supplementary Table S2). This region was $\sim 2-\mathrm{Mb}$ long on the Bm_scaf33. Next, we performed gene prediction analysis for the candidate region using gene prediction models in KAIKOBLAST and we found 77 candidate genes (data not shown). For the Ws mutation, we delimited the locus to 100-kb-long regions between 2445094 (nscaf2829-50F) and 2541315 (nscaf2829-39R) on the Bm_scaf33 using $\sim 400 \mathrm{BC}_{1}$ individuals (Figure 2 and Supplementary Table S2). However, there were no candidate genes within this region (data not shown). According to the linkage analysis of the $B m$ gene, although the mapping procedure used $\sim 1800 \mathrm{BC}_{1}$ individuals, the $\mathrm{Bm}$-linked region could not be narrowed down further within an $\sim 2-\mathrm{Mb}$-long region on the Bm_scaf33, thereby suggesting suppression of recombination. Therefore, the $B m$-narrowed region was wider than that of Ws (Figure 2).

\section{Recombination analysis between $\mathrm{Bm}$ and $\mathrm{Ws}$}

To confirm the recombination between $B m$ and $W s$, the moth phenotype was observed in seven egg batches obtained from the cross between wild type $\left(++^{B m} /+^{B m} ;+{ }^{W s} /+^{W s}\right)$ females with $F_{1}$ males $\left(B m\right.$ female $\times W s$ male $\left(B m /+{ }^{B m} ;+{ }^{W s} / W s\right)$ ) (Supplementary Figure S1 and Table 2). Among 1163 individuals obtained from 7 batches, none of the normal type expected as recombinants appeared (Table 2). The segregation ratio between $B m$ phenotype and $W s$ phenotype was $\sim 1: 1$ (Table 2). These strongly suggested that recombination did not occur between $\mathrm{Bm}$ and $\mathrm{Ws}$ alleles (Table 2), although the genetic distance between both genes is $14.7 \mathrm{cM}$ in the linkage map of $B$. mori.

\section{FISH analysis}

To confirm the possibility of suppression of crossing over, we performed FISH analysis of the pachytene nuclei of the wild type (p50) and Bm mutant (No. 908) using four BACs mapped on Bm_scaf33 and a BAC on Bm_scaf92. FISH analysis revealed that the five $\mathrm{BAC}$ probes mapped onto p50 in a sequence according to the KAIKObase information. However, the FISH signals between 4D3C (yellow) and 1G10A (red) were invertedly ordered in No. 908 (Figure 3 and Table 1). Therefore, a chromosomal inversion is apparent in No. 908. This chromosome feature explained why recombination was not observed between $\mathrm{Bm}$ and $\mathrm{Ws}$ loci.

Comparative genomic analysis of the $B m$ and $W s$ regions, and other lepidopteran genomes

Based on the comparative genomic analysis, we found that the $B m$ and Ws regions shared synteny with a region associated with wing- and body-colour variations in different lepidopteran species of $B$. betularia and Heliconius butterflies (Joron et al., 2006; Kronfost et al., 2006; Papa et al., 2008; Ferguson et al., 2010; van't Hof et al., 2011). The carbonaria region, which determines the phenotype of industrial melanism in $B$. betularia, shared synteny with the upstream region of B. mori genetic linkage group 17, corresponding to 2390014
Table 2 Segregation of moth phenotype in the cross normal $(+/+,+/+)$ females $\times B m$ and $W s(+/ B m,+/ W s)$ males

\begin{tabular}{|c|c|c|c|}
\hline \multirow[t]{2}{*}{ Batch numbers } & \multicolumn{3}{|c|}{ Moth phenotype } \\
\hline & $\begin{array}{c}\text { 'Bm }(+/ B m,+/+)^{\prime} \text { and } \\
\text { 'Bm and } W s(+/ B m,+/ W s)^{\text {'b }}\end{array}$ & $\begin{array}{c}\text { 'Ws } \\
(+/+,+W s)^{\prime}\end{array}$ & $\begin{array}{l}\text { 'Normal } \\
(+/+,+/+)\end{array}$ \\
\hline 1 & 101 & 83 & 0 \\
\hline 2 & 86 & 97 & 0 \\
\hline 3 & 80 & 73 & 0 \\
\hline 4 & 87 & 94 & 0 \\
\hline 5 & 29 & 50 & 0 \\
\hline 6 & 81 & 104 & 0 \\
\hline 7 & 100 & 98 & 0 \\
\hline
\end{tabular}

The female body colour was not clear and both phenotypes could not be distinguished. anly male was used for screening of the phenotypes.

${ }^{b} B m(+/ B m,+/+)$ and $B m$, and $W s(+/ B m,+/ W s)$ phenotypes could not be judged whether it was only $B m$ or $B m$ and Ws phenotype, because the majority of the phenotypes had black wings.



Figure 3 Inversion in chromosome 17 of $B$. mori No. 908 strain carrying the Black moth loci. The inverted order of FISH signals between 4D3C (yellow) and 1 G10A (red) is apparent compared with the p50 strain. BAC codes are shown in the same colours as the signals. Marker sequence (see Yasukochi et al. 2006) or GenBank accession numbers for the BACs are shown on the left of the black bar, which represents $B$. mori chromosome 17 drawn to a relative scale in $\mathrm{Mb}$ taken from KAIKObase. White and black scale bars represent $5 \mu \mathrm{m}$ and $5 \mathrm{Mb}$ for the bivalents and chromosome 17 , respectively. See Table 1 for details of the BAC probe information.

2875682 on the Bm_scaf33 (between trehalase $1 B$ and $\operatorname{lrtp}$ ) (Figure 4) (van't Hof et al., 2011). This phenotype is very similar to the $B m$ phenotype. The Ws region shared synteny with $H$. melpomene linkage group 15 and this region was located between the $H$. melpomene yellow hindwing bar $(\mathrm{Hm} \mathrm{Yb})$ and $\mathrm{H}$. melpomene hindwing margin (HmSb) candidate regions, corresponding to $2880220-2568710$ and 2195 440-2 281515 on the Bm_scaf33, respectively ( $H m Y b$, between BGIBMGA005665 and 005652; HmSb, between BGIBMGA005650 and 005559) (Figure 4). Both of these causal genes determine wing colour variations in $H$. melpomene (Ferguson et al., 2010). In addition, this region overlapped with the mimetic patterning regions, $\mathrm{Yb}, \mathrm{P}$ and $\mathrm{Cr}$, in other Heliconius species, that is, $H$. cydno, $H$. erato and $H$. numata (Joron et al., 2006; Kronfost et al., 2006; Papa et al., 2008). These 


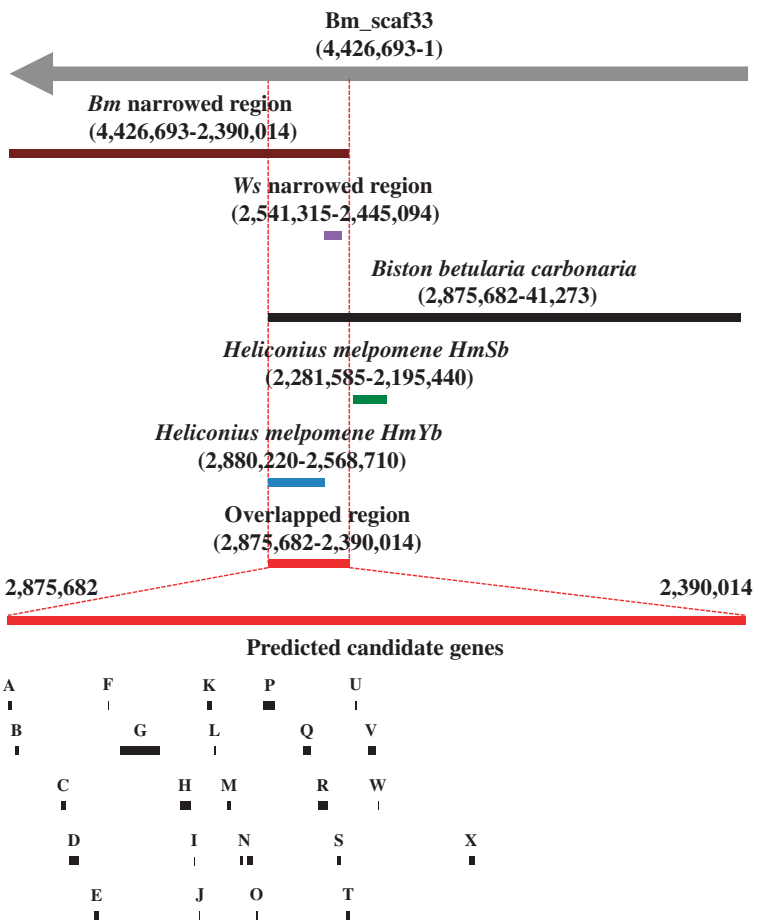

Figure 4 Localisation of $B m$ and $W s$ regions narrowed down to the Bm_scaf33 and their positional relationships with candidate regions of carbonaria, HmSb and HmYb. The brown, purple, black, blue and green bars indicate the $B m, W s$, carbonaria, HmSb and Hmyb regions, respectively. The red bar indicates the overlapping region for all genes. The carbonaria gene determines the phenotype of industrial melanism in the British peppered moth, B. betularia (van't Hof et al., 2011). The HmSb and HmYb genes exhibit phenotypes with a hindwing margin and a yellow hindwing bar in H. melpomene, respectively (Ferguson et al., 2010). Lower bars indicate 25 predicted genes. A, BGIBMGA005665; B, 005664; C, 005663; D, 005548; E, 005662; F, 005661; G, 005549; H, 005660; I, 005550; J, 005659; K, 005551; L, 005658; M, 005552; N, 005553 and 005554; O, 005555; P, 005556; Q, 005657; R, 005656; S, 005557; T, 005655; $\mathrm{U}, 005558 ; \mathrm{V}, 005654 ; \mathrm{W}, 005653$; and $\mathrm{X}, 005652$. The letters correspond to Supplementary Figure S3 and Suppementary Table S3.

results suggest that this region may control wing- and body-colour variations in lepidopteran insects. Therefore, we focused on the predicted genes within the overlapping candidate regions of five genes, that is, Bm, Ws, carbonaria, $\mathrm{HmYb}$ and $\mathrm{HmSb}$ (Figure 4), and we performed gene expression analysis based on the RT-PCR results.

\section{RT-PCR analysis of candidate genes in the overlapping region}

Using KAIKObase, we predicted $24 \mathrm{Bm}$ and $W s$ candidate genes within the overlapping region: BGIBMGA005665 (A), 005664 (B), 005663 (C), 005548 (D), 005662 (E), 005661 (F), 005549 (G), 005660 $(\mathrm{H}), 005550$ (I), 005659 (J), 005551 (K), 005658 (L), $005552(\mathrm{M})$, 005553 and $005554(\mathrm{~N}), 005555(\mathrm{O}), 005556(\mathrm{P}), 005657(\mathrm{Q}), 005656$ (R), $005557(\mathrm{~S}), 005655(\mathrm{~T}), 005558(\mathrm{U}), 005654(\mathrm{~V}), 005653(\mathrm{~W})$ and 005652 (X) (Figure 4 and Table 3). First, we investigated whether these candidate genes were expressed in the forewing from pupal day 0 to adult day 0 (Supplementary Figures S2 and S3). RT-PCR analysis demonstrated that seven candidate genes were expressed in the forewing, that is, BGIBMGA005550 (I), 005658 (L), 005552 (M), 005657 (Q), 005656 (R), 005557 (S) and 005655 (T) (Supplementary Figure S3 and Table 3). In particular, three candidate genes, that is, BGIBMGA005658 (L), $005657(\mathrm{Q})$ and 005655 (T), exhibited clear differences in their expression profiles where these genes were properly expressed only in the wild-type strain (p50T) (Figure 5, Supplementary Figure S3 and Table 3). In the genomic PCR analysis using primer sets for these three differentially expressed genes, identical PCR products were obtained from respective genes in p50T, No. 908 and u42 individuals. These results suggest that the differences in the expression profiles were not due to the primerbinding sites but the expression levels (data not shown). Next, we cloned and sequenced four additional candidate genes, that us, BGIBMGA005550 (I), $005552(\mathrm{M}), 005656$ (R) and 005557 (S), and compared their sequences in the wild type (p50T), Bm mutant (No. 908) and Ws mutant (u42). According to the KAIKObase database search, these genes correspond to the full-length cDNA or expressed sequence tag clones AK383524; FS895121, FS917714 and FY019022; AK38029 and FY026966; and AK384540 and FY030309, respectively (Table 3). Therefore, we prepared primer sets based on the $5^{\prime}$ - and 3 'untranslated regions using the sequences of each expressed sequence tag clone and performed RT-PCR analyses. Two candidate genes, that is, BGIBMGA005550 (I) and 005656 (R), lacked mutations in the coding regions (Supplementary Figure S4) and we could not detect the transcripts of two candidate genes, BGIBMGA005552 (M) and 005557 (S) (Supplementary Figure S4). Overall, the results of the PCR and sequencing analysis suggest that BGIBMGA005658 (L), 005657 (Q) and 005655 (T) may be candidates for the $B m$ and $W s$ genes.

\section{DISCUSSION}

In this study, we attempted to isolate two genes responsible for moth colour mutations, that is, $B m$ and $W s$, based on positional cloning using $B$. mori genome information. The genetic and genomic analysis demonstrated the following: (i) the candidate regions of the $\mathrm{Bm}$ and $W s$ genes are located in $\sim 2-\mathrm{Mb}$-long and 100-kb-long regions on the same scaffold Bm_scaf33 of chromosome 17; (ii) chromosome 17 of $\mathrm{Bm}$ mutation harbours inversion within a compartment corresponding to Bm_scaf33; and (iii) the Bm and Ws regions share synteny with a region associated with wing- and body-colour variations in different lepidopteran species (Joron et al., 2006; Kronfost et al., 2006; Papa et al., 2008; Ferguson et al., 2010; van't Hof et al., 2011). Based on our results, we hypothesise that this common region may control wingand body-colour variations in lepidopteran insects. These results provide insights into the molecular mechanisms that control colour variations in Lepidoptera.

Chikushi (1960) mapped the $B m$ gene to $0.0 \mathrm{cM}$ on $B$. mori genetic linkage group 17 based on three-point crosses using the $B m$, ow and bts genes (Chikushi, 1960). In addition, Doira et al. (1981) reported that the $W s$ gene was located at $14.7 \mathrm{cM}$ in the same linkage group based on recombination analysis between the Ws and bts genes. FISH analysis demonstrated that a proximal region of chromosome 17 in No. 908 has an inversion. Thus, no recombination among $1163 \mathrm{BC}_{1}$ individuals is most probably caused by suppression of chromosome crossing over. Taking into account for classical linkage analysis, similar pattern of gene expression results in the present study and recent finding for mimicry and pheromone response (Joron et al. 2011; Nishikawa et al. 2015; Wadsworth et al. 2015), inversion-associated mutation is a possible explanation for $\mathrm{Bm}$ origin. This supposes the $B m$ and $W s$ share a mechanism for regulating wing and body colouration. However, the classical recombination value was calculated by a combination of different cross-experiments (Chikushi, 1960, Doira et al. 1981). Hence, it is also possible to predict the Bm locates in the proximity to $W s$ as well as any position in $\sim 2-\mathrm{Mb}$ region in Bm_scaf33. 


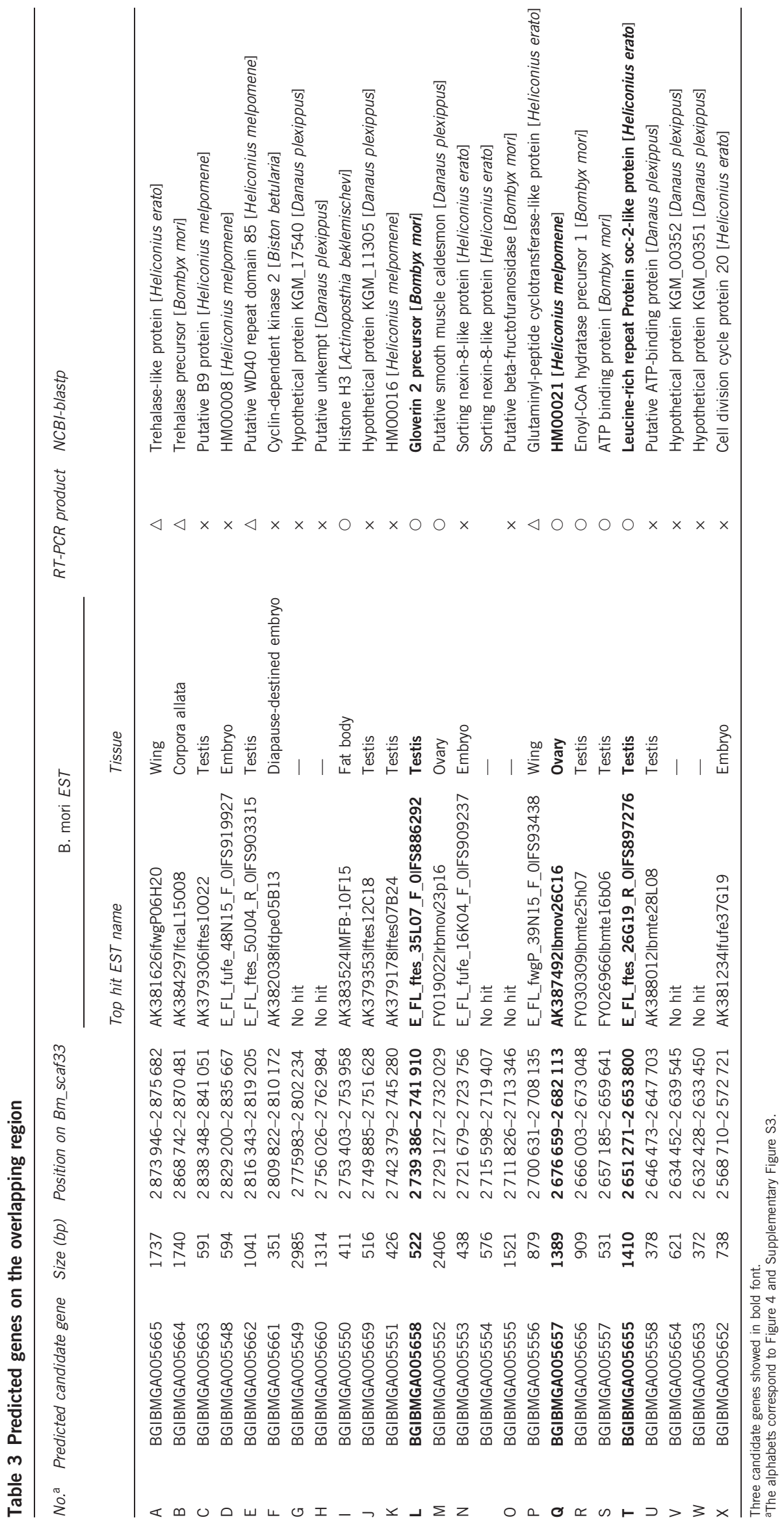




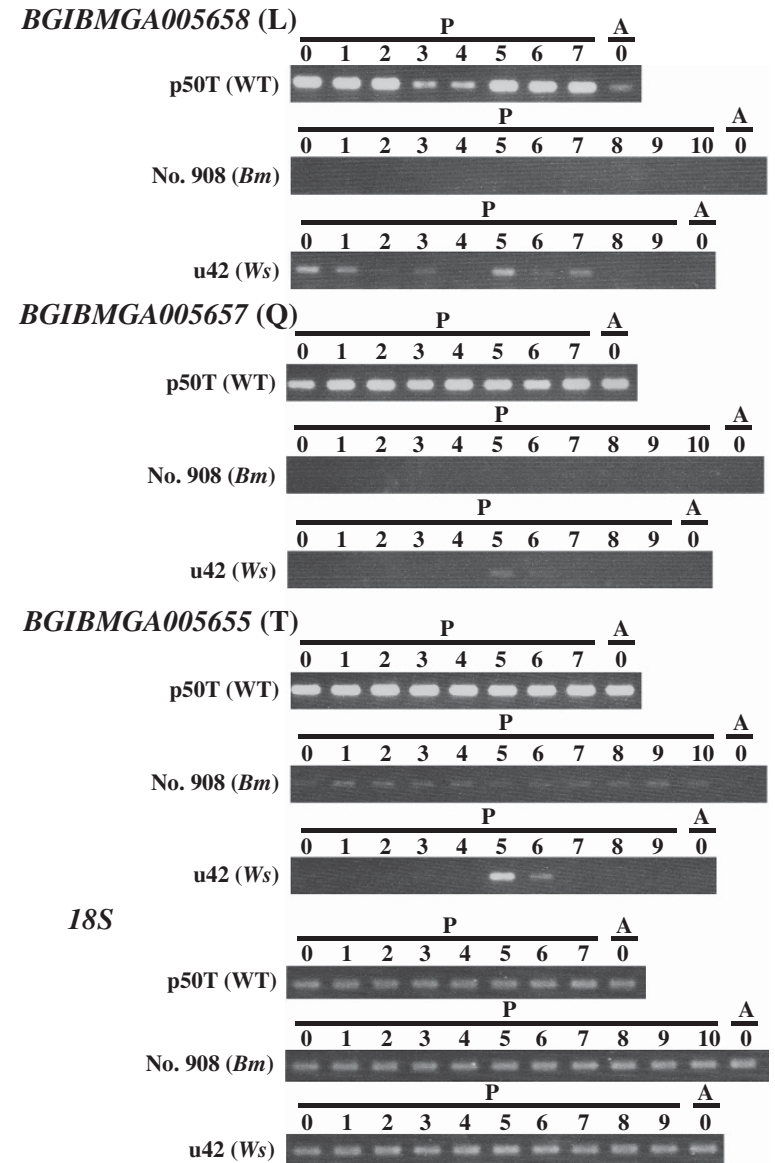

Figure 5 RT-PCR analysis of the candidate genes of $B m$ and Ws. Stagespecific expression profiles of three candidate genes, BGIBMGA005658 (L), $005657(\mathrm{Q})$ and $005655(\mathrm{~T})$, were investigated with p50T (wild type), No. 908 ( $B m$ mutant) and $u 42$ (Ws mutant) strains. $P$ and $A$ indicate pupa and adult, respectively. The numbers under the $P$ and $A$ bars show the day for each stage. $18 S$ ribosomal RNA was used as an internal control.

According to the linkage analysis of the Ws gene, we narrowed down $W s$ to a 100-kb-long region on the Bm_scaf33; however, there was no candidate gene within this region. Thus, the following two hypotheses are proposed. First, the nucleotide responsible for Ws mutation may correspond to a cis-regulatory element of $W s$, which controls $W s$ expression in the spot at the apex of the wing. Second, the candidate gene may exist in an unknown genomic region that is specific to the mutant strain. This may explain why we could not find the candidate gene, because it was predicted using the genome sequence of the model strains p50T and Dazao, which exhibits the wild-type phenotype (International Silkworm Genome Consortium, 2008). Therefore, we are currently attempting to determine the genome sequence of the Ws mutant strain and B. mandarina by shotgun sequencing analysis.

RT-PCR analysis of the predicted genes indicated that three genes, that is, BGIBMGA005658 (L), $005657(\mathrm{Q})$ and $005655(\mathrm{~T})$, are current candidates for the $\mathrm{Bm}$ and $\mathrm{Ws}$ genes. The expression profiles of these genes revealed that transcripts were detected only in the wild-type strain ( $\mathrm{p} 50 \mathrm{~T})$, thereby suggesting that the phenotypes may be due to functional inactivation of these genes via haploinsufficiency or dominant-negative mutations. Investigations of the expression profiles of these genes using $F_{1}$ individuals will help to identify the gene responsible for these mutations. In addition, further gene expression analysis using RNA-seq and microarray will help to identify the genes responsible for $\mathrm{Bm}$ and Ws. Furthermore, BGIBMGA005658 encodes the gloverin 2 precursor in $B$. mori; however, it is not present at the orthologous location in Heliconius (Ferguson et al., 2010). This may be because of a difference in genome information between Bombyx and Heliconius. In general, gloverins have been reported to be antibacterial proteins in lepidopteran insects because of their antibacterial activity against Escherichia coli, Gram-positive bacteria, fungi and viruses (Kawaoka et al., 2008; Yi et al., 2013). Therefore, the possibility the gloverin 2 precursor is candidates for $B m$ and $W s$ genes will be a low.

The candidate regions of $\mathrm{Bm}$ and $\mathrm{Ws}$ genes shared synteny with a region associated with wing- and body-colour variations in different lepidopteran species (Joron et al., 2006; Kronfost et al., 2006; Papa et al., 2008; Ferguson et al., 2010; van't Hof et al., 2011). The phenotypes of the Bm and Ws mutations comprise black scales on the moth body and a spot at the apex of wing, respectively. The colour of both mutants is mainly black; however, the coloured parts of the body differ from each other. In the carbonaria type of $B$. betularia, the phenotype has a black body colour, which is very similar to the $B m$ mutation. However, the $\mathrm{HmSb}, \mathrm{HmYb}, \mathrm{Cr}, \mathrm{P}$ and $\mathrm{Yb}$ genes of Heliconius species are associated with mimetic patterning of the wings. The wing colouration is consistent with the phenotype of the Ws mutation. These results suggest that the control of colour pattern formation in lepidopterans may have a common genetic basis, although the critical factor has yet to be identified. Further studies to clarify the nature of this regulation will help to understand the molecular mechanisms that regulate the development of wing colouration.

\section{Data archiving}

The B. mori linkage maps and genetic markers used for genotyping are available from http://www.shigen.nig.ac.jp/silkwormbase/index.jsp and http://sgp.dna.affrc.go.jp/KAIKObase/.

\section{CONFLICT OF INTEREST}

The authors declare no conflict of interest.

\section{ACKNOWLEDGEMENTS}

We thank Mr Munetaka Kawamoto (University of Tokyo) for technical assistance and Dr Yutaka Banno (National BioResource Project (NBRP), Kyushu University) for providing the silkworm strains. This research was supported by grants from MAFF-NIAS (Agrigenome Research Program), MEXT (KAKENHI No. 22128004), NBRP (National BioResource Project) and JST (Professional Program for Agricultural Bioinformatics), Japan.

Banno Y, Yamamoto K, Nishikawa K, Tamura K, Yamamoto K, Aso Y (2010). Integration of the twenty-fourth and twenty-seventh linkage groups of the silkworm Bombyx mori. I Insect Biotech Sericol 79: 67-70.

Chikushi H (1960). A new linkage group of the silkworm, Bombyx mori. J Seric Sci Jpn 29 278 in Japanese.

Dai FY, Qiao L, Tong XL, Cao C, Chen P, Chen J et al. (2010). Mutations of an arylalkylamine- $N$-acetyltransferase, $B m$-iAANAT, are responsible for silkworm melanism mutant. J Biol Chem 285: 19553-19560.

Diora H, Kihara H, Masuda S (1981). Linkage analysis of the 'Wild wing spot' gene in the silkworm. Proc Sericult Sci Kyushu 12: 64 (in Japanese).

Ferguson L, Lee SF, Chamberlain N, Nadeau N, Joron M, Baxter S et al. (2010). Characterization of a hotspot for mimicry: assembly of a butterfly wing transcriptome to genomic sequence at the $\mathrm{HmYb} / \mathrm{Sb}$ locus. Mol Ecol 19: 240-254.

Goldsmith MR, Shimada T, Abe H (2005). The genetics and genomics of the silkworm Bombyx mori. Annu Rev Entomol 50: 71-100.

Hasimoto H (1961). Genetika studo pri melanismo ce morusa silkraŭpo, Bombyx mori. J Seric Sci Jpn 30: 389 (in Japanese with Esperanto summary).

Heliconius Genome Consortium (2012). Butterfly genome reveals promiscuous exchange of mimicry adaptations among species. Nature 487: 94-98. 
International Silkworm Genome Consortium (2008). The genome of a lepidopteran model insect, the silkworm Bombyx mori. Insect Biochem $\mathrm{Mol}$ Biol 38: 1036-1045.

Ito K, Katsuma S, Yamamoto K, Kadono-Okuda K, Mita K, Shimada T (2009). A 25 bp-long insertional mutation in the BmVarp gene causes the waxy translucent skin of the silkworm, Bombyx mori. Insect Biochem Mol Biol 39: 287-293.

Ito K, Katsuma S, Yamamoto K, Kadono-Okuda K, Mita K, Shimada T (2010). Yellow-e determines the color pattern of larval head and tail spots of the silkworm Bombyx mori. J Biol Chem 285: 5624-5629.

Ito K, Kidokoro K, Sezutsu H, Nohata J, Yamamoto K, Kobayashi I et al. (2008). Deletion of a gene encoding an amino acid transporter in the midgut membrane causes resistance to a Bombyx parvo-like virus. Proc Natl Acad Sci USA 105: 7523-7527.

Joron M, Papa R, Beltrán M, Chamberlain N, Mavárez J, Baxter S et al. (2006). A conserved supergene locus controls color pattern diversity in Heliconius butterflies. PLoS Biol 4: e303.

Joron M, Frezal L, Jones RT, Chamberlain NL, Lee SF, Haag CR et al. (2011). Chromosomal rearrangements maintain a polymorphic supergene controlling butterfly mimicry. Nature 477: 203-206.

Kanbe R, Nara S (1959). Gemetical studies of a new mutamt, white bamded black $(w b)$ wing, in silkworm. J Seric Sci Jpn 28: 37 (in Japanese).

Kawaoka S, Katsuma S, Daimon T, Isono R, Omuro N, Mita K et al. (2008). Functional analysis of four Gloverin-like genes in the silkworm Bombyx mori. Arch Insect Biochem Physiol 67: 87-96.

Kronforst MR, Kapan DD, Gilbert LE (2006). Parallel genetic architecture of parallel adaptive radiations in mimetic Heliconius butterflies. Genetics 174: 535-539.

Nishikawa H, lijima T, Kajitani R, Yamaguchi J, Ando T, Suzuki Y et al. (2015). A genetic mechanism for female-limited Batesian mimicry in Papilio butterfly. Nat Genet 47 405-409.

Niwa R, Namiki T, Ito K, Shimada-Niwa Y, Kiuchi M, Kawaoka S et al. (2010). Non-molting glossy/shroud encodes a short-chain dehydrogenase/reductase that functions in the 'Black Box' of the ecdysteroid biosynthesis pathway. Development 137: 1991-1999.

Papa R, Morrison CM, Walters JR, Counterman BA, Chen R, Halder G et al. (2008). Highly conserved gene order and numerous novel repetitive elements in genomic regions linked to wing pattern variation in Heliconius butterflies. BMC Genomics 9: 345

Parchem RJ, Perry MW, Patel NH (2007). Patterns on the insect wing. Curr Opin Genet Dev 17: 300-308.
Sahara K, Marec F, Traut W (1999). TTAGG telomeric repeats in chromosomes of some insects and other arthropods. Chromosome Res 7: 449-460.

Sahara K, Yoshido A, Shibata F, Fujikawa-Kojima N, Okabe T, Tanaka-Okuyama M et al. (2013). FISH identification of Helicoverpa armigera and Mamestra brassicae chromosomes by BAC and fosmid probes. Insect Biochem Mol Biol 43: 644-653.

Shibata F, Sahara K, Naito Y, Yasukochi Y (2009). Reprobing of multicolour FISH in preparations of lepidopteran chromosomes. Zoolog Sci 26: 187-190.

van't Hof AE, Edmonds N, Dalíková M, Marec F, Saccheri IJ (2011). Industrial melanism in British peppered moths has a singular and recent mutational origin. Science 332 : 958-960.

Wadsworth CB, Li X, Dopman EB (2015). A recombination suppressor contributes to ecological speciation in OSTRINIA moths. Hered Adv online 114: 593-600.

Yamamoto T (1986). Linkage group of the black-striped pupal wing gene in the silkworm. Proc J Seric Sci Jpn 56: 60 (in Japanese).

Yamamoto K, Nohata J, Kadono-Okuda K, Narukawa J, Sasanuma M, Sasanuma S et al. (2008). A BAC-based integrated linkage map of the silkworm Bobmyx mori. Genome Biol 9: R21.

Yasukochi Y, Ashakumary LA, Baba K, Yoshido A, Sahara K (2006). A second generation integrated map of the silkworm reveals synteny and conserved gene order between lepidopteran insects. Genetics 173: 1319-1328.

Yoshido A, Bando H, Yasukochi Y, Sahara K (2005). The Bombyx mori karyotype and the assignment of linkage groups. Genetics 170: 675-685.

Yoshido A, Sahara K, Yasukochi Y (2014). Chapter 6; Silkmoths (Lepidoptera). In: Sharakhov IV (ed). Protocols for Cytogenetic Mapping of Arthropod Genomes. CRC Press: London, pp 219-256.

You M, Yue Z, He W, Yang G, Xie M, Zhan D et al. (2013). A heterozygous moth genome provides insights into herbivory and detoxification. Nat Genet 45: 220-225.

Yi HY, Deng XJ, Yang WY, Zhou CZ, Cao Y, Yu XQ (2013). Gloverins of the silkworm Bombyx mori: structural and binding properties and activities. Insect Biochem $\mathrm{Mol} \mathrm{Biol} \mathrm{43:}$ 612-625.

Zhan S, Guo Q, Li M, Li M, Li J, Miao X et al. (2010). Disruption of an N-acetyltransferase gene in the silkworm reveals a novel role in pigmentation. Development 137: 4083-4090.

Zhan S, Merlin C, Boore JL, Reppert SM (2011). The monarch butterfly genome yields insights into long-distance migration. Cell 147: 1171-1185.

Supplementary Information accompanies this paper on Heredity website (http://www.nature.com/hdy) 